\title{
Capsule endoscopy in the diagnosis of an exophytic gastrointestinal stromal tumor in the small intestine of a young adult woman: A case report
}

\author{
XIAOLING XU ${ }^{1}$, ZHENGLONG $\mathrm{CAO}^{2}$ and HAIHANG ZHU ${ }^{1}$ \\ ${ }^{1}$ Department of Gastroenterology, Subei People's Hospital, Clinical Medical School, Yangzhou University Affiliated Hospital, \\ Yangzhou, Jiangsu 225001; ${ }^{2}$ Department of Gastroenterology, Tianchang Hospital \\ of Traditional Chinese Medicine, Tianchang, Anhui 239300, P.R. China
}

Received May 20, 2015; Accepted July 22, 2015

DOI: $10.3892 / \operatorname{mco} .2015 .697$

\begin{abstract}
Gastrointestinal stromal tumors (GISTs) are mesenchymal tumors that mainly arise in the gastrointestinal tract. They are usually asymptomatic and are incidentally discovered during endoscopy or surgery. Diagnosis is confirmed by histological examination of the specimen. This is the case report of an asymptomatic GIST of the small intestine diagnosed by wireless capsule endoscopy. The tumor was initially suspected to be a leiomyoma, as GISTs in young adults are rare and are mainly discovered incidentally during colorectal cancer screening. The patient was a 35-year-old woman with occult gastrointestinal bleeding, with a normal medical history. An endoscopic assessment of the upper and lower GI tract (gastroscopy and colonoscopy) was performed, but did not reveal any abnormalities. Subsequently, an exophytic tumor initially suspected as leiomyoma or external pressure was detected in the small intestine by capsule endoscopy. A computed tomography scan was suggestive of a soft tissue tumor arising from the small intestine. A surgical specimen was obtained and the immunohistochemical examination revealed that the tumor was positive for CD117 and discovered on GIST-1 markers, while the markers of carcinoma, melanoma and lymphoma were negative, which was consistent with a diagnosis of a low-risk GIST with a mitotic count of $<5 / 50$ high-power fields. In this study, we aimed to present in detail the capsule endoscopic and radiological characteristics, as well as the findings of the histological examination of the surgical specimen. In conclusion, when occult blood is detected in the stool, even when gastroscopy and colonoscopy
\end{abstract}

Correspondence to: Professor Haihang Zhu, Department of Gastroenterology, Subei People's Hospital, Clinical Medical School, Yangzhou University Affiliated Hospital, 98 West Nantong Road, Yangzhou, Jiangsu 225001, P.R. China

E-mail: zhuhaihang@medmail.com.cn

Key words: capsule endoscopy, exophytic tumor, small intestine gastrointestinal stromal tumor, obscure gastrointestinal bleeding, young adult woman reveal no abnormal findings, small intestinal lesions should be suspected. Exophytic small intestinal GISTs, although rare, particularly in younger patients, they should be considered by physicians in the differential diagnosis of obscure GI bleeding of unknown origin, in order to reduce morbidity and mortality. Capsule endoscopy may be considered to be the first choice as a diagnostic tool for obscure GI bleeding.

\section{Introduction}

Gastrointestinal stromal tumors (GISTs) are common mesenchymal tumors of the gastrointestinal tract, which arise from the interstitial cells of Cajal (ICCs). GISTs are rare tumors, comprising $0.1-0.3 \%$ of all GI malignancies (1).

The majority of GISTs are located in the stomach (60-70\%) and the small intestine (20-25\%). GISTs are difficult to diagnose and early diagnosis may be challenging. A definitive diagnosis requires immunohistochemical confirmation. These tumors have a known potential for malignancy and their prognosis is poor when the mitotic index is high.

GI bleeding is the most common presentation (50\%) of GISTs and is usually associated with ulceration of the tumor into the intestinal lumen (2).

This is the report of an unusual case of an exophytic GIST originating from the small bowel, which was asymptomatic, apart from a weakly positive occult blood test in a young adult woman. At first, the occult blood was attributed to hemorrhoids. However, the patient had a family history of colon cancer; therefore, she required more thorough investigation, despite the lack of obvious symptoms. GISTs in the small intestine may be identified by capsule endoscopy following negative upper endoscopy and colonoscopy findings and are confirmed through histological examination of the surgical specimen.

\section{Case report}

A 35-year-old woman presented with a weakly positive occult blood test, following a regular health screening. The patient had no abdominal discomfort, weight loss or any other clinical manifestations; however, she had a family history of colon 


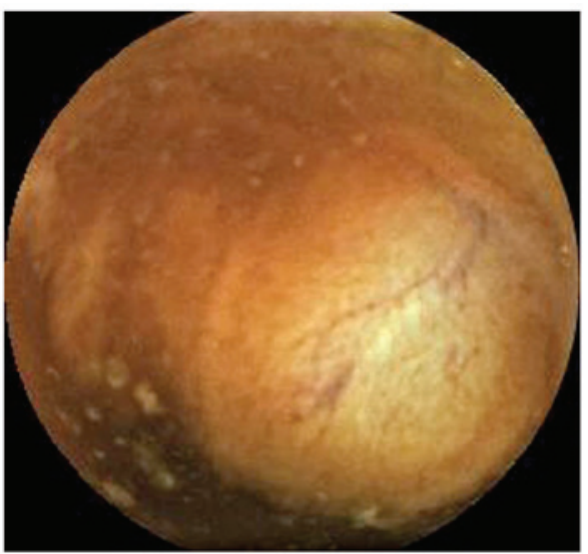

Figure 1. Capsule endoscopic visualization of a submucosal tumor with a smooth surface.

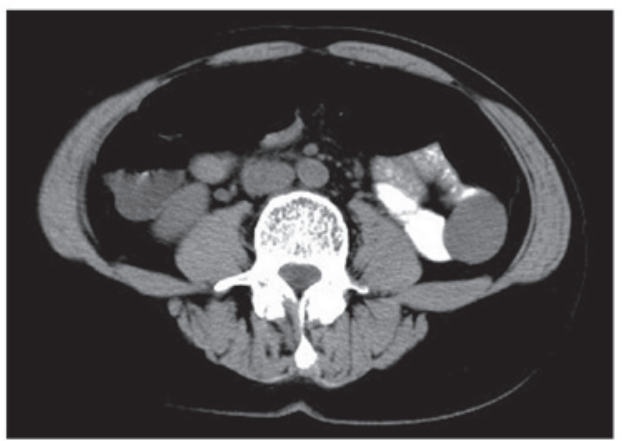

Figure 2. Computed tomography scan revealed a smooth outlined solid mass.

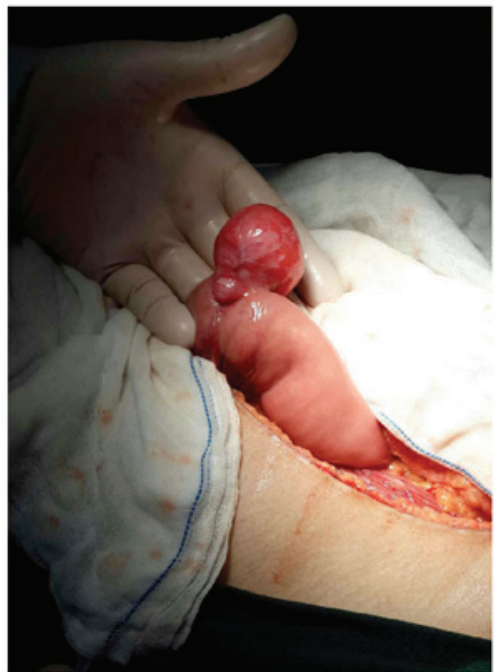

Figure 3. A pedunculated exophytic tumor arising from the ileum was identified intraoperatively.

cancer. The patient's blood pressure was $117 / 86 \mathrm{mmHg}$, her heart rate was 80 beats $/ \mathrm{min}$, and her temperature $36.9^{\circ} \mathrm{C}$. There was no anemia on examination.

An endoscopic assessment of the upper and lower GI tract (gastroscopy and colonoscopy) was performed, but did not reveal any abnormal findings. Subsequently, an exophytic tumor was identified in the small intestine by capsule endos-

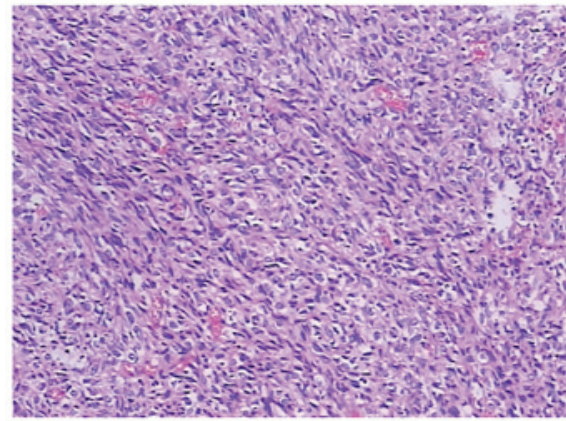

Figure 4. Epithelioid differentiation with perinuclear and cytoplasmic vacuolization. Hematoxylin and eosin stain; magnification, x100.

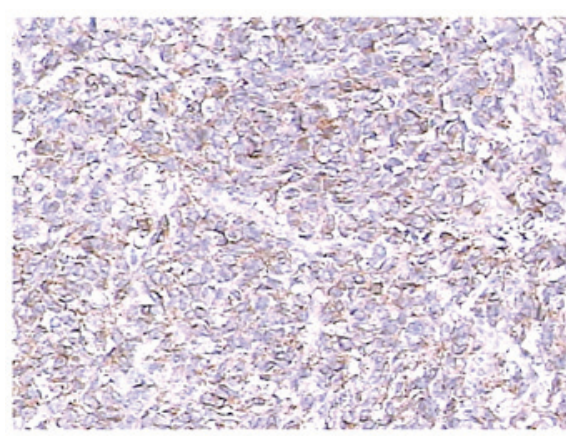

Figure 5. c-Kit proto-oncogene (CD117) strong and diffuse cytoplasmic staining; magnification, $\mathrm{x} 100$.

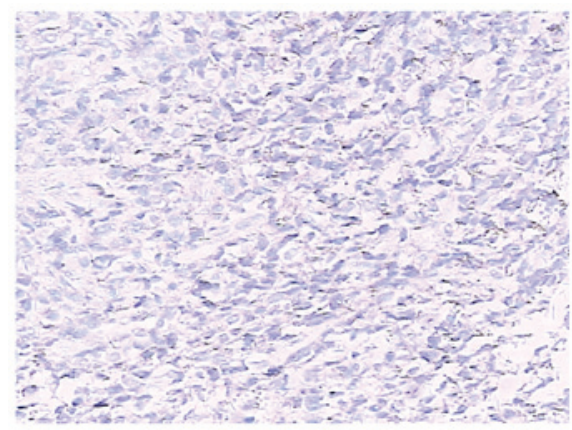

Figure 6. Strong immunoreactivity for discovered on GIST-1; magnification, x100.

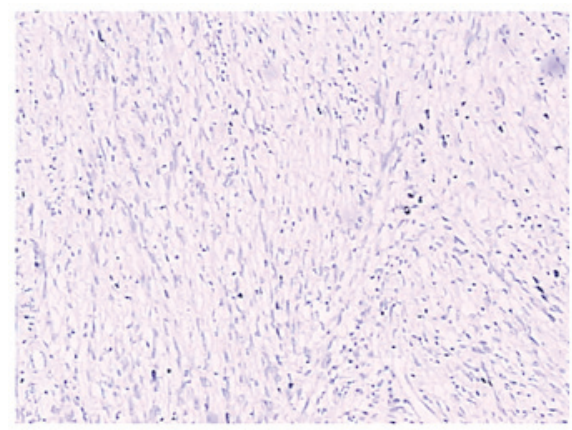

Figure 7. Ki-67 low proliferation index (2\%); magnification, x100

copy (Fig. 1). A computed tomography scan revealed a mass sized $1.5 \times 2.0 \times 2.5 \mathrm{~cm}$ in the lower left quadrant (Fig. 2). An enhanced computed tomography scan revealed a smoothly 
outlined hypervascular solid mass. The computed tomography scan was suggestive of a soft tissue tumor arising from the small intestine. Based on the capsule endoscopic and radiological findings, a diagnosis of a small intestinal mass was established. The patient was subsequently referred for surgery and underwent laparotomy. The intraoperative findings included an isolated exophytic pedunculated mass, sized $1.5 \times 2.0 \times 2.5 \mathrm{~cm}$, on the wall of the mid-ileum (Fig. 3). There was no macroscopic evidence of distant spread. The histopathological report was that of a GIST arising from the small intestine (Fig. 4). On pathological examination of the surgical specimen, the tumor was established as low-risk according to the Fletcher's criteria, with a mitotic index of $<5$ mitoses $/ 50$ high-power fields (HPFs) (3). The immunohistochemistry results revealed a high expression of CD117 (Fig. 5) and discovered on GIST-1 (DOG-1) (Fig. 6); however, desmin, S100 and pancytokeratin were negative. The Ki-67 expression revealed a low proliferative index (2\%) (Fig. 7).

\section{Discussion}

GISTs are mesenchymal tumors of the GI tract that express the tyrosine kinase receptor and originate from the ICCs. GISTs arise from the muscularis mucosa or muscularis propria layers and mainly exhibit an endophytic growth pattern, which may present with evidence of obstruction or bleeding. Exophytic tumors present as a large mass lesion or, if perforation has occurred, with symptoms of peritonitis (4).

The diagnosis is confirmed by histological examination. Immunohistochemistry is crucial for differentiating GISTs from leiomyomas and neurogenic tumors (5). Specific abnormalities of c-Kit (CD117) and platelet-derived growth factor receptor $\alpha$ (PDGFRA) are the main oncogenic event in GISTs. Approximately $75-80 \%$ of GISTs harbor gain-of-function c-Kit mutations, 7-10\% harbor gain-of-function PDGFRA mutations, and the remaining are defined as wild-type, as they have no c-Kit or PDGFRA mutations $(6,7)$.

In this study, immunohistochemistry was conclusive in determining the histology of the tumor, based on CD117 and DOG-1 positivity, resulting in the final diagnosis of small intestinal GIST.

For GISTs, the main symptoms are bleeding and obstruction, which is caused by the growing tumor. Pressure necrosis and ulceration of the overlying mucosa may cause GI bleeding. In the present case, a weakly positive occult blood test was the most important finding beyond atypical abdominal discomfort. The GI bleeding was only obscure, as there was no obvious necrosis or ulceration of the overlying mucosa on capsule endoscopy. This case was found incidentally during a colorectal cancer screening. As the patient had a family history of colon cancer, endoscopic examination was suggested. Subsequently, an exophytic GIST arising from the small intestine was identified.

GISTs may be classified as low- or high-risk tumors by taking into consideration the possibility of metastasis or recurrence based on the macroscopic/histological findings. GISTs have a known potential for malignancy and their prognosis is poor when the mitotic index is high. The poor prognostic predictive factors include tumor size $>5 \mathrm{~cm}$, mitotic index $>5 / 50$ HPFs, tumor necrosis, and a Ki-67 (MIB-1) index of $>10 \%$, and are associated with high mortality $(2,8)$.

Taking into consideration the prognostic predictive factors found in our case, such as the small size of the tumor $(2.5 \mathrm{~cm})$, low mitotic index $(<5 / 50 \mathrm{HPFs})$, absence of necrosis and a $\mathrm{Ki}-67$ (MIB-1) index of $<10 \%$, there is little chance of recurrence of the tumor. Surgical resection is the only effective therapy and recommended for nearly all adult patients.

GISTs are mesenchymal tumors of the GI tract that typically occur in adults aged $>40$ years, whereas they are rare in in younger ( $<40$ years) patients (4). Studies on GISTs in young adult patients are limited due to their rarity. However, as our patient was a 34-year-old woman, GI stromal tumors should be included in the differential diagnosis of intestinal mesenchymal tumors presenting as a single mass in young female adults, despite diagnostic physical findings not being readily apparent.

Wireless capsule endoscopy is considered to be the first-line non-invasive diagnostic tool of the small intestine for patients with obscure GI bleeding, enabling early diagnosis and application of a definitive therapy, thereby increasing the chance of survival.

Although GISTs of the small intestine presenting with obscure GI bleeding in young adult women are a rare finding, clinicians should consider capsule endoscopy even following negative findings on upper endoscopy and colonoscopy.

\section{References}

1. Badshah MB, Riaz H, Korsten MA, Dhala A, Park YH, Abadi M and Badshah MB: Gastro-intestinal stromal tumor (GIST) complicating a colonic interposition: A novel case report. BMC Res Notes 7: 604, 2014

2. Fletcher CD, Berman JJ, Corless C, Gorstein F, Lasota J, Longley BJ, Miettinen M, O'Leary TJ, Remotti H, Rubin BP, Shmookler B, Sobin LH and Weiss SW: Diagnosis of gastrointestinal stromal tumors: a consensus approach. Hum Pathol 33: 459-465, 2002

3. Misawa S, Takeda M, Sakamoto H, Kirii Y, Ota H and Takagi H: Spontaneous rupture of a giant gastrointestinal stromal tumor of the jejunum: A case report and literature review. World J Surg Oncol 12: 153, 2014.

4. Sezer A, Yagci MA, Hatipoglu AR, Coskun I, Cicin I, Usta U and Temizoz O: A rare cause of intestinal obstruction due to an exophytic gastrointestinal stromal tumor of the small bowel. Signa Vitae 4: 32-34, 2009.

5. Manxhuka-Kerliu S, Sahatciu-Meka V, Kerliu I, Juniku-Shkololli A, Kerliu L, Kastrati M and Kotorri V: Small intestinal gastrointestinal stromal tumor in a young adult woman: A case report and review of the literature. J Med Case Rep 8: 321, 2014.

6. Hirota S, Isozaki K, Moriyama Y, Hashimoto K, Nishida T, Ishiguro S, Kawano K, Hanada M, Kurata A, Takeda M, et al: Gain-of-function mutations of c-kit in human gastrointestinal stromal tumors. Science 279: 577-580, 1998.

7. Nannini M, Biasco G, Di Scioscio V, Di Battista M,Zompatori M, Catena F, Castellucci P, Paterini P, Dei Tos AP, Stella F, et al: Clinical, radiological and biological features of lung metastases in gastrointestinal stromal tumors (case reports). Oncol Rep 25: 113-120, 2011.

8. Emory TS, Sobin LH, Lukes L, Lee DH and O'Leary TJ: Prognosis of gastrointestinal smooth-muscle (stromal) tumors: Dependence on anatomic site. Am J Surg Pathol 23: 82-87, 1999. 